\title{
Communication jonctionnelle et interactions neuro-glio-vasculaires
}

(CIRB)

\section{Christian Giaume}

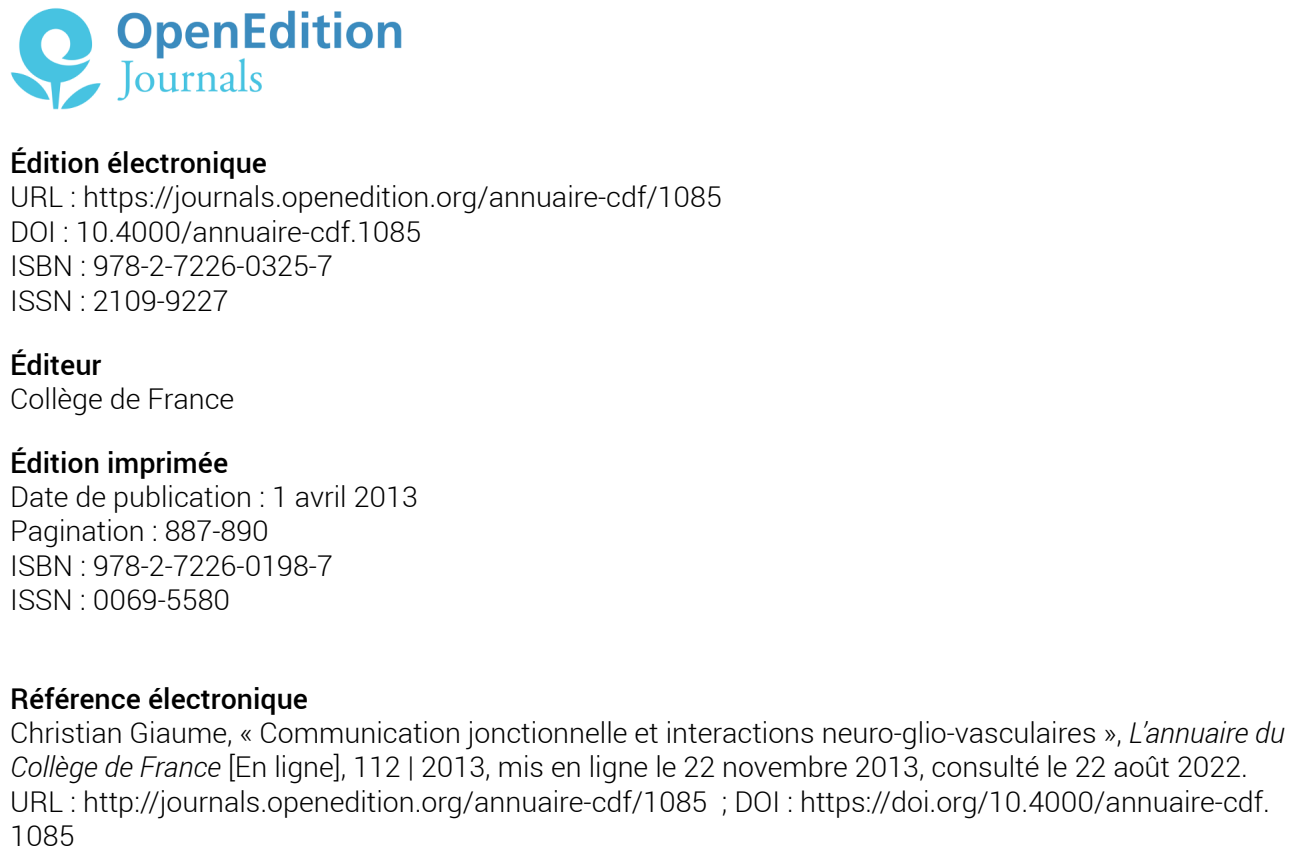

Tous droits réservés 


\title{
Communication jonctionnelle et interactions neuro-glio-vasculaires (CIRB)
}

\author{
Responsable : Christian GIAUME
}

Dans le système nerveux central, les connexines, protéines constituantes des jonctions communicantes (JCs) ou gap junctions et des hémicannaux, sont exprimées en grande quantité dans les cellules gliales, en particulier dans les astrocytes. Les travaux de notre équipe portent principalement sur différents aspects des propriétés et des rôles de ces protéines dans les interactions que les cellules gliales établissent avec les neurones et le système vasculaire que ce soit dans des conditions physiologiques ou pathologiques.

\section{RECHERCHE}

\section{Régulation de l'expression et de la fonction des connexines astrocytaires lors de perturbations du cycle veille-sommeil}

(Xinhe LIU, Pascal EZAN)

Les astrocytes sont des importants modulateurs de l'activité neuronales par le biais de plusieurs mécanismes comme par exemple : la capture de neurotransmetteurs, l'homéostasie potassique ou l'apport de substrats énergétiques, ce qui implique qu'ils contribuent de manière dynamique aux fonctions centrales, en particulier le cycle veille-sommeil. Les astrocytes forment des réseaux de cellules communicantes grâce à un niveau élevé d'expression de connexines, les constituants moléculaires des jonctions communicantes. Dans un premier temps nous avons étudié les effets de drogues connues pour modifier l'état d'éveil chez la souris sur le couplage entre astrocytes du cortex somatosensoriel. Nous avons observé que le Modafinil $(100 \mu \mathrm{M})$, un agent psychostimulant, augmente $(+25 \%)$ la communication jonctionnelle mesurée par la technique de «dye coupling »; cet effet dépend de l'activité neuronale puisqu'il n'est pas observé en présence de TTX. À l'opposé le GHB (acide gamma-hydroxybutyrique ; $1 \mathrm{mM}$ ), un psychotrope dépresseur, diminue $(-25 \%)$ ce couplage intercellulaire; cet effet ne dépend pas de l'activité neuronale car il est observé en présence de TTX. Ces données pharmacologiques indiquent que des drogues ayant des effets opposés sur l'état de vigilance ont des effets contraires sur la communication jonctionnelle. D'autre part, en collaboration avec le laboratoire du Pr P. Magistretti (EPFL, Lausanne) nous avons étudié les effets d'une privation de sommeil sur l'expression et la fonction de communication des connexines astrocytaires. Quatre groupes expérimentaux correspondant à : (i) des souris privées de sommeil pendant $6 \mathrm{~h}$ sans récupération, (ii) des souris privées de sommeil mais ayant récupérées pendant $3 \mathrm{~h}$, (iii) des souris contrôles sacrifiées au même point horaire que les souris (i), et (iv) des souris contrôles sacrifiées au même point horaire que les souris (ii). Le dosage des ARNm codant pour les Cx43 et $\mathrm{Cx} 30$ indique une augmentation du messager pour la $\mathrm{Cx} 30$ après privation de sommeil. Il est intéressant de remarquer que l'induction de la Cx30 reste encore élevée après $3 \mathrm{~h}$ de récupération ce qui n'est pas le cas pour des gènes des astrocytes impliqués dans le métabolisme tel que la PTG (protein targeting to glycogen) par 
exemple. Des données préliminaires indiquent que ces augmentations se retrouvent également au niveau de l'expression de la protéine et correspondent à une augmentation du couplage jonctionnel $(+25 \%)$. Prise dans leur ensemble ces observations démontrent que l'expression et la fonction de la Cx30 sont modifiées lorsque le cycle veille-sommeil est perturbé.

\section{Bases moléculaires des fonctions des connexines astrocytaires dans le système nerveux central}

(Martine CoHEn-Salmon, Anne-Cécile Boulay, Silvia BuRBassi)

Les astrocytes, cellules gliales majoritaires du cerveau, jouent un rôle actif dans la physiologie vasculaire cérébrale en interagissant de façon dynamique avec les vaisseaux sanguins au moyen de ramifications, ou pieds astrocytaires, qui couvrent la totalité du système vasculaire cérébral. Ils participent ainsi non seulement à l'induction et au maintien de l'intégrité de la barrière hémato-encéphalique (BHE), mais aussi au contrôle du flux sanguin, et au transfert des métabolites du sang vers les neurones. Nous avons récemment montré que les $\mathrm{Cx} 30$ et $\mathrm{Cx} 43$ sont particulièrement enrichies au niveau périvasculaire aux points de contact entre pieds astrocytaires. De plus, nous avons établi que leur absence, chez une souris dont les gènes codant pour ces protéines ont été inactivés, entraine une fragilisation de la BHE qui devient perméable lorsque la pression vasculaire augmente. Ces deux connexines sont donc nécessaires à l'intégrité de la BHE. Nos travaux se concentrent maintenant sur le rôle de $\mathrm{Cx} 30$ et $\mathrm{Cx} 43$ dans le maintien de la BHE, en adressant les changements morphologiques et moléculaires induits dans le système vasculaire cérébral par leur inactivation.

Dans ce but, nous développons de nouveaux outils moléculaires pour étudier les fonctions des connexines astrocytaires. Les connexines possèdent des fonctions d'adhésion et de signalisation intracellulaire indépendantes de leurs fonctions canal (hémicanaux et jonctions gap). Les outils moléculaires d'inactivation des connexines astrocytaires actuellement disponibles ne permettent pas de cibler ces différentes fonctions. Nous avons développé des constructions lentivirales à tropisme astrocytaire exprimant des formes mutées de Cx30 et Cx43 entrainant la formation de canaux fermés. Leur utilisation nous permettra prochainement d'adresser plus spécifiquement les fonctions des connexines astrocytaires dans la neurotransmission et dans les régulations vasculaires cérébrales.

\section{Connexines astrocytaires et maladie d'Alzheimer}

(Annette KoulaKofF, Chenju YI, Edwige AmIGOu)

Dans diverses pathologies cérébrales, aiguës ou chroniques, un remodelage morpho-fonctionnel des astrocytes est observé. Il s'accompagne le plus souvent de modifications de l'expression et des fonctions des connexines. Nous nous sommes attachés à étudier cette question dans le contexte de la maladie d'Alzheimer (MA). Nous avons analysé l'expression des connexines astrocytaires dans des coupes de cerveau humain provenant de biopsies post-mortem de patients atteints de la maladie d'Alzheimer (MA) par une approche immunohistochimique. Ces coupes sont situées dans le lobe temporal du cortex, l'une des régions où des dépôts de peptide $\beta$-amyloide (A $\beta)$ s'accumulent et forment des plaques séniles, lésions 
histopathologiques caractéristiques de cette pathologie. Nous avons confirmé la présence d'augmentation de l'immunoréactivité de la $\mathrm{Cx} 43$ dans les astrocytes réactifs au niveau des dépôts de peptides $\mathrm{A} \beta$ et mis en évidence des augmentations de Cx30 bien que plus faibles et moins fréquentes. La qualité des coupes n'a cependant pas permis une quantification des résultats, comme cela avait été fait précédemment chez des souris APP/PS1, modèles murins de la MA. En revanche, les diminutions d'immunoréactivité des connexines associées à une petite proportion de plaques chez les souris APP/PS1 n'ont pas été décelées dans les coupes humaines par comparaison au tissu sain environnant.

Afin d'analyser l'incidence de l'absence des connexines astrocytaires sur divers aspects de la pathologie que développent les souris APP/PS1, nous produisons des souris APP/PS1 dans lesquels les gènes des connexines astrocytaires sont innactivés (KO). La production de souris APP/PS1/KOCx43 (i.e. Cx43f1/1 /GFAPcre) vient d'être lancée. Les souris APP/PS1/KOCx30 sont maintenant disponibles et leur analyse a été initiée cette année. La cinétique d'apparition des plaques séniles et la production des deux peptides $\beta$-amyloides qui les composent, $A \beta 40$ et $A \beta 42$, ont été comparées chez les souris APP/PS1 et les souris APP/PS1/KOCx30. Dans les deux génotypes, les premières plaques sont décelables vers 4-5 mois. Chez des souris plus âgées (9 et 16 mois) leur abondance et distribution sont comparables. En accord avec ces observations, aucune différence significative n'a été observée dans la teneur en $A \beta 40$ et en A $\beta 42$, testée par ELISA dans des extraits de cerveaux de souris âgées de 4, 9 et 16 mois. Par conséquent l'invalidation de la Cx30 n'affecte pas l'accumulation de peptides A $\beta$ chez la souris APP/PS1.

Ces souris ont également été utilisées pour analyser la contribution relative des deux connexines astrocytaires à l'activité hémicanal qui a été décelée dans les astrocytes réactifs associés aux plaques amyloïdes. L'activation des hémicanaux, voies d'échange entre le cytoplasme et le milieu extracellulaire, a été évaluée dans des tranches de cerveaux de souris APP/PS1 et de APP/PS1/KOCx30, en utilisant un test de capture de bromure d'éthidium. Des résultats semblables ont été obtenus dans les deux génotypes, ce qui indique qu'une activation des hémicanaux persiste en absence de $\mathrm{Cx} 30$. Cette observation concorde avec les données pharmacologiques montrant qu'un peptide mimétique bloquant sélectivement la Cx43 (Gap26) inhibe les hémicanaux aussi efficacement qu'un bloquant plus général des hémicanaux (ions $\mathrm{La}^{++}$) et suggère que l'on peut écarter une contribution de la Cx30 à l'activité hémicanal dans les astrocytes.

\section{PUBLICATIONS}

Orellana J.A., von Bernhardi R., Giaume C. et Sáez J.C., « Glial hemichannels and their involvement in aging and neurodegenerative diseases », Review in the Neurosciences, 23, 2012, 163-177.

Arama J., Boulay A.C., Bosc C., Delphin C., Loew D., Rostaing P., Amigou E., Ezan P., Wingertsmann L., Guillaud L., Andrieux A., Giaume C. et Cohen-Salmon M., «Bmcc1s, a novel brain-isoform of Bmcc1, affects cell morphology by regulating MAP6/STOP functions », PLoS One, 7, 2012, e35488.

Ezan P., André P., Cisternino S., Saubaméa B., Boulay A.C., Doutremer S., Thomas M.A., Quenech'du N., Giaume C. et Cohen-Salmon M., « Deletion of astroglial connexins weakens 
the blood-brain barrier », Journal of Cerebral Blood Flow and Metabolism, 4 avril 2012 [doi: $10.1038 / \mathrm{jcbfm}]$.

Giaume C., Orellana J.A., Abudara V. et Sáez J.C., «Connexin-based channels in astrocytes: how to study their properties », Methods in Molecular Biology, 814, 2012, 283303.

Koulakoff A., Mei X., Orellana J.A., Sáez J.C. et Giaume C., « Glial connexin expression and function in the context of Alzheimer's disease », Biochimica Biophysica Acta, 1818, 2012, 2048-2057.

Roux L., Benchenane K., Rothstein J.D., Bonvento G. et Giaume C., «Plasticity of astroglial networks in olfactory glomeruli », Proceeding National Academy Sciences (USA), 108, 2011, 18442-18446.

Giaume C. et Liu X., «From a glial syncytium to a more restricted and specific glial networking », Journal of Physiology (Paris), 106, 2012, 34-39.

Tencé M., Ezan P., Amigou E. et Giaume C., «Increased interaction of connexin43 with zonula occludens-1 during inhibition of gap junctions by $\mathrm{G}$ protein-coupled receptor agonists », Cell Signaling, 24, 2012, 86-98.

Pannasch U., Vargová L., Reingruber J., Ezan P., Holcman D., Giaume C., Syková E. et Rouach N., « Astroglial networks scale synaptic activity and plasticity », Proceeding National Academy Sciences (USA), 108, 2011, 8467-8472.

Orellana J.A., Shoji K.F., Abudara V., Ezan P., Amigou E., Sáez P.J., Jiang J.X., Naus C.C., Sáez J.C. et Giaume C., «Amyloid $\beta$-induced death in neurons involves glial and neuronal hemichannels », Journal of Neuroscience, 31, 2011, 4962-4977.

Orellana J.A., Froger N., Ezan P., Jiang J.X., Bennett M.V., Naus C.C., Giaume C. et Sáez J.C., «ATP and glutamate released via astroglial connexin 43 hemichannels mediate neuronal death through activation of pannexin 1 hemichannels », Journal of Neurochemistry, 118, 2011, 826-840.

\title{
Communication intercellulaire et infections microbiennes UMR 7241 / U 1050 (CIRB)
}

\author{
Responsable : Guy Tran VAN NHIEU
}

\section{TRAVAUX EFFECTUÉS (2011-2012)}

Les travaux de l'équipe visent à comprendre le mécanisme d'invasion des cellules épithéliales par Shigella, l'agent de la dysenterie bacillaire. Les sections développées ci-dessous ne concernent qu'une partie des activités de l'équipe. Nous avons plus récemment étendu notre champ d'étude au mécanisme de virulence des Escherichia coli enteropathogène (EPEC) en étudiant la régulation de l'activité de leur T3SS, ainsi qu'à celui du pneumocoque (Streptoccus pneumoniae), une bactérie pathogène responsable de maladies invasives dont des méningites. 